ORIGINAL ARTICLE

\title{
Clinical and laboratory evaluation of compliance in acute lymphoblastic leukaemia
}

\author{
B M de Oliveira, M B Viana, C L Zani, A J Romanha
}

Arch Dis Child 2004;89:785-788. doi: 10.1136/adc.2003.030775 See end of article for
authors' affiliations

Correspondence to: Prof. B M de Oliveira, Departamento de Pediatria da Faculdade de Medicina da UFMG, Av Alfredo Balena 190, Belo

Horizonte 30130-100,

Brazil;

benigna@uol.com.br

Accepted

12 November 2003

\begin{abstract}
Aim: To evaluate compliance in children with acute lymphoblastic leukaemia (ALL).
Methods: Compliance was assessed through specific interviews, annotations from medical charts, and erythrocytic determination of 6-mercaptopurine metabolites.

Results: A total of 39 patients who had concluded maintenance phase of chemotherapy were included in the study. Mothers were responsible for delivering 6-MP in $87 \%$ of cases. Thirty five interviewees said that medical prescription was well understood and that the main reason for non-compliance was forgetfulness. Non-compliance was detected through interviews (33.3\% of the cases), reports from medical charts $(30.7 \%)$, and drug determination (16.6\%); $53.8 \%$ of children were found to be non-compliant. Noncompliance was significantly associated with chronic undernourishment. Although not statistically significant, there was a trend for the group of non-compliant children to be associated with low per capita family income. No significant associations of non-compliance with age at diagnosis, gender, parents' schooling level, number of family members, power consumption, and medians of absolute leucocyte or neutrophil blood counts were detected. A short follow up period precluded valid analysis on outcome. In the non-compliant group $(n=21)$, seven children relapsed, contrasting with three relapses in the compliant group $(n=18)$.

Conclusions: Results suggest that non-compliance is one of the mechanisms which underlies the adverse influence of socioeconomic factors on the outcome of children with ALL. Additional studies are necessary to confirm this hypothesis. Comprehensive approaches to the problem of non-compliance are urgently needed.
\end{abstract}

A cute lymphoblastic leukaemia (ALL) is the most common malignancy in childhood. Nowadays, 70$80 \%$ of the recently diagnosed children in developed countries present a disease free survival of over five years, and most will eventually be cured. ${ }^{1}$

Results of several ALL treatment groups in childhood in developing countries have been inferior to those obtained in developed countries, even when aggressive treatment protocols were used. This has been ascribed to an interaction of a set of adverse factors, such as poor performance of the health system, racial differences, malnutrition and, more recently, low socioeconomic status. ${ }^{2}$

Another noteworthy hypothesis is the possibility of treatment non-compliance. ${ }^{3}$ Families in low cultural and socioeconomic conditions presumably have greater difficulty in understanding the disease and properly following medical instructions and prescriptions. Therefore, the conduct of studies evaluating compliance as a factor related to chemotherapy response and, consequently, to the prognosis of patients with ALL, is highly important, mainly in communities with contrasting socioeconomic, ethnic, and cultural conditions. To date, few studies have aimed at studying ALL compliance, ${ }^{4-15}$ on which the present investigation is centred.

\section{PATIENTS AND METHODS}

From October 1996 to December 2000, 91 patients with ALL, aged less than 18 years, were diagnosed at the Federal University of Minas Gerais (UFMG), Brazil; 65 had started the maintenance phase of the Brazilian therapeutic protocol GBTLI-93. Thirty nine children had concluded the maintenance phase of chemotherapy before the endpoint of 6mercaptopurine (6-MP) metabolite assay and were considered for the study. Only for these children were the sequential levels of 6-MP metabolites considered adequate for compliance analysis. Table 1 summarises patient characteristics. The follow up period ranged from 16.5 to 82.9 months (median 63) until September 2003, and was complete for all patients.

ALL was diagnosed through bone marrow smear stained with May-Grünwald-Giemsa and confirmed by immunophenotyping.

Patients were stratified into three relapse risk groups based on age and white blood cell count at diagnosis. Maintenance phase in the three groups consisted of oral 6-MP in an initial dosage of $50 \mathrm{mg} / \mathrm{m}^{2} /$ day (maximum $100 \mathrm{mg} / \mathrm{m}^{2} /$ day) and intramuscular methotrexate (MTX) in an initial dosage of 25 $\mathrm{mg} / \mathrm{m}^{2} /$ week (maximum $40 \mathrm{mg} / \mathrm{m}^{2} /$ week). Dosages were adjusted in order to maintain leucocyte count between $2 \times 10^{9}$ and $3 \times 10^{9}$ per litre and phagocyte count above $0.5 \times 10^{9}$ per litre. Patients were instructed to take medication at night. For lower and standard risk groups the maintenance phase began in the 22nd week of treatment and for higher risk group in the 77 th week. Patients were randomly allocated into two groups: two versus one and a half years of maintenance duration. Medications were available to all patients free of charge.

Two nutritional indices were evaluated at diagnosis: weight-for-age (WAZ) and height-for-age (HAZ), expressed as standard deviation $\mathrm{Z}$ score, in relation to the National Centre for Health Statistics population. ${ }^{16}$ Since the prevalence of malnutrition in Brazil is higher than in developed countries, the cut-off point $\mathrm{Z}=-1.28$ (centile 10) was adopted to define individual malnutrition, since $Z=-2$, as recommended by $\mathrm{WHO}^{17}$ is more specific but less sensitive.

Abbreviations: 6-MP, 6-mercaptopurine; 6-TGN, 6-thioguanine; ALL, acute lymphoblastic leukaemia; HAZ, height-for-age Z score; MMP, methylmercaptopurine; MTX, methotrexate; WAZ, weight-for-age Z score 
Table 1 Characteristics of the studied population

\begin{tabular}{ll}
\hline Characteristics & Patients ( $\mathbf{n}=39$ ) \\
\hline Gender (M/F) & $14 / 25$ \\
Median (variation) of age at diagnosis (in years) & $4.8(1.5-16.3)$ \\
Median (variation) of white cell count at diagnosis (×109/) & $5.6(0.7-272)$ \\
Median (variation) of weight-for-age Z score & $-0.31(-2.09-1.79)$ \\
Median (variation) of height-for-age Z score & $-0.41(-1.71-1.94)$ \\
Standardised prevalence of undernourishment measured by weight-for-age Z score ${ }^{35}$ & $12.5 \%$ \\
Standardised prevalence of undernourishment measured by height-for-age Z score & $10.8 \%$ \\
Median (variation) of family size (in number of individuals) & $4(3-13)$ \\
Median (variation) of household power consumption (KWh/day) & $4.9(0-15.08)$ \\
Mean (variation) of family per capita income (minimum wages/month) & $0.74(0.17-9.62)$ \\
Number (percentage) of children with pre-B CD10 positive leukaemia & $29(74 \%)$ \\
\hline
\end{tabular}

All families were interviewed. Questions concerned identification of residents in the patient's household; schooling level; employed individuals, profession, occupation, and individual monthly income expressed in minimum wages/ month (from these data per capita family income was calculated); household description, power consumption calculated through power invoice in KWh/day; complementary data such as eating habits, leisure, cultural activities, family medical assistance, and family decision making process.

Compliance was evaluated through three methods: specific interviews, annotations in medical charts, and monitoring of intra-erythrocyte concentration of 6-MP metabolites.

Specific interviews were scheduled twice in the maintenance phase: 8 weeks after its beginning, and 8-12 weeks before the end of the treatment or at the time of relapse. Questions included medications taken by the child at that time; whether there was an individual responsible for regular medication delivery; regularity of medication intake time; frequency of failure in receiving 6-MP and/or MTX without medical instruction to interrupt chemotherapy; and understanding of medical instructions.

Patients had their medical charts reviewed by the same investigator who recorded all assistant physician's written reports on incorrect or irregular usage of 6-MP and/or MTX, and chemotherapy interruptions without medical advice.

Blood samples for the assay of 6-MP metabolites were collected from the eighth week of maintenance phase onwards, with an approximate interval of eight weeks. Patients should be under continuous administration of 6MP for a minimum period of four weeks and should have not received packed red cell transfusion in the six weeks preceding blood collection. The minimum number of samples prescribed for each group of patients was defined according to the length of the maintenance phase, ranging from two to six samples. Red cells were prepared according to the method described by Lennard. ${ }^{18}$ Nucleotides of 6-thioguanine (6TGN) and methylmercaptopurine (MMP) were separated and quantified through high performance liquid chromatography, employing a methodology combining thiopurine extraction as recommended by Dervieux and Boulieu ${ }^{19}$ and analysis proposed by Lennard and Singleton. ${ }^{20}$

Definition of non-compliance was specific for each method: (1) interviews: acquiescence by the interviewees that the child failed to receive the medication twice or more during the maintenance phase, without medical instruction to do so; (2) medical charts: detection by the investigator that 6-MP delivery was interrupted or decreased without medical authorisation for at least two weeks; two or more records of irregular administration of 6-MP; (3) significant and simultaneous decrease of 6-TG and MMP concentration in relation to other samples, without any decrease in the prescribed 6-MP dosage in the previous four weeks.
Association of non-compliance with categorical variables was tested through either $\chi^{2}$ (corrected by Yates) or Fisher's exact tests. Association of non-compliance with continuous variables was evaluated through the Mann-Whitney test. Associations were tested defining non-compliant children as those who were detected either through any of three methods or through at least two methods combined. In the nutritional analysis, calculations were done using Epi Info, version $6.03 .{ }^{21}$ In all statistic tests the two tailed significance level of $\mathrm{p}=0.05$ in relation to alpha error was adopted.

The UFMG Research Ethics Committee approved the study. Authorisation for study enrolment was obtained through written informed consent of the patient's parents or legal guardians, who were informed about the study aims. They were unaware of whether a regular blood sample would be separated for metabolite determination.

\section{RESULTS}

Ten of the 39 patients relapsed; eight died after complications following relapse. The most frequent site of relapse was the bone marrow (9/10).

Eighty two per cent of the interviews were conducted with the patients' mothers. All interviewees indicated that the patient used 6-MP and 37 (95\%) that 6-MP was administered at night. The mothers were responsible for medication intake in $34(87 \%)$ of the cases. In the two cases in which 6-MP was not always administered by the same person, patients proved to be non-compliant. Additionally, two adolescents were responsible for their own medication intake. The schooling level of the person responsible for regular medication delivery for treatment compliance ranged from 1 to 15 years (median 6). Thirty five families $(89.7 \%)$ reported that medical instructions were always understood, indicating that occasional doubts concerned more the disease itself and childcare than information about schedule and adverse effects of drugs. Interviews indicated that $13 / 39$ patients (33.3\%) were non-compliant. The interviewees indicated that the main reason for not following the prescription was forgetfulness, mostly because of housework, or religious or social activity.

In $12 / 39$ charts $(30.7 \%)$, irregular or incorrect dosage or interruption of drugs without medical advice was recorded. The median of leucocyte and neutrophil counts throughout the maintenance phase ranged from 2.55 to $7.0 \times 10^{9}$ per litre (median $3.6 \times 10^{9}$ per litre) and $1.1 \times 10^{9}$ per litre to $3.9 \times 10^{9}$ per litre (median $1.7 \times 10^{9}$ per litre), respectively.

Metabolite assay was done in 36/39 subjects for a total of 183 samples; in three cases the minimum number of two samples could not be obtained for analysis. Intra-erythrocyte concentration of 6-TGN and MMP ranged from 0 to 1934.1 $\mathrm{pmol} / 8 \times 10^{8}$ red cells (median 319.7 ) and 0 to $45.0 \mathrm{nmol} /$ $8 \times 10^{8}$ red cells (median 6.3 ), respectively. Non-compliance was detected in six cases (16.6\%), five of which were also considered non-compliant through other methods (table 2). 
Table 2 Number of non-compliant children according to the $\operatorname{method}(\mathrm{s})$ for detection of compliance

\begin{tabular}{ll}
\hline Methods & Non-compliant children \\
\hline Metabolite assay + interview + chart & 2 \\
Metabolite assay + interview & 2 \\
Metabolite assay + chart & 1 \\
Interview + chart & 3 \\
Only metabolite assay & 1 \\
Only interview & 6 \\
Only chart & 6 \\
Total & 21
\end{tabular}

Complete absence of the metabolites in one of the blood samples was noted in only one patient.

Twenty one of 39 patients $(53.8 \%)$ were considered noncompliant through at least one method, and were compared to the remaining 18 compliant children. There was a significant association $(\mathrm{p}=0.045)$ between non-compliance and undernourishment (HAZ $<-1.28)$. Although not statistically significant $(p=0.15)$, there was a trend for the monthly per capita income of the non-compliant group (median of 0.63 minimum wage/month) to be inferior to that of the compliant group (median of 1 minimum wage/month). There was no association between non-compliance and age at diagnosis $(p=0.53)$, gender $(p=0.97)$, schooling level of individual alleged responsible for medication intake $(p=0.77)$, number of family members $(p=0.73)$, daily household power consumption $(p=0.84)$, median white cell count $(\mathrm{p}=1)$, and neutrophils $(\mathrm{p}=0.6)$.

In eight cases $(20.5 \%)$ non-compliance was detected through at least two methods (table 2). Comparing these with the remaining patients, there was no association between non-compliance and other variables, including HAZ $(p=0.32)$, and per capita income $(p=0.21)$.

Due to an insufficient follow up period, analyses of outcome were considered inadequate at this moment. However, among 21 non-compliant patients as defined previously, seven relapsed, six of whom died. In four cases the relapse occurred after the conclusion of chemotherapy, and in three cases, during the maintenance phase. In the group of 18 compliant children, three relapsed. In one case, relapse occurred after the conclusion of chemotherapy, and in the two others, in the 8th and 15th weeks of maintenance phase, both of whom died. The remaining patients were alive and in remission in September 2003.

\section{DISCUSSION}

Compliance is a complex and multifaceted subject that plays an important part in clinical practice and medical research. It can be assessed through direct methods (drug levels in biological samples) or indirect methods (patient or parental reports, physician opinion, pill count, etc).22 Although many methods are available, none are entirely valid or reliable. ${ }^{23}$ Therefore, combination of methods is a valid way of increasing sensitivity for the detection of non-compliance. ${ }^{24}$ Since non-compliance is potentially harmful to the outcome of sick children, we consider that sensitivity is more important than specificity. This balance in the present study was achieved by defining non-compliance in two ways.

There is a consensus that treatment compliance is mainly influenced by the patient and/or his or her parents' understanding of the illness. For some authors, the patient has a greater tendency to comply with the treatment, if he or she believes that the physician is right, that the illness is hazardous to his or her health, and that the treatment will reduce the odds of complications and death..$^{25}$ Since children diagnosed with ALL, reaching remission, become practically asymptomatic and always remain under extended and complex treatment, non-compliance is somehow expected. ${ }^{4}$

The suitable level of compliance needed for the cure of ALL, or inversely, a non-compliance ratio that could result in relapse, is unknown. ${ }^{15}$ British researchers have suggested that a child bearing ALL should receive at least $95 \%$ of the prescribed medication to be considered compliant. ${ }^{3}$ Studies found in specialised literature have used different methodologies for compliance evaluation, hampering any comparisons. Nonetheless, results of the present study are similar to those of other reports on children and adolescents with ALL, considered non-compliant in $2-52 \%$ of cases,${ }^{4-15}$ being higher in adolescents. ${ }^{458}$ In the present study, however, there was no association between patient age and non-compliance, albeit only six patients were aged 10 years or older.

The conduct of interviews with patients and their parents is the most widely used method in clinical practice for compliance evaluation. It is surprising that interviews conducted in the eighth week of the maintenance phase disclosed that $18 \%$ of the patients had already skipped medication intake at least once during this short time period. These occurrences could still be understated, given that selfreports of non-compliance with medical prescriptions are generally more reliable than self-reports of compliance. Additionally, if either family or patient admits in an interview that failure occurred at least twice in a long period of time, it is possible that the event might have been more frequent. Similarly, medical evaluations usually underestimate the frequency of non-compliance. ${ }^{22}$

6-TGN and MMP are products of two competitive metabolic pathways, for which an inverse correlation was expected. The reasons for observing individual variations in the ability to produce 6-MP metabolites are as yet unclear. One is the difference in the activity of the enzyme thiopurine methyltranferase (TPMT). TPMT favours the formation of MMP, which leads to a reduction in 6-TGN formation through competitive metabolic pathways. The existence of a genetic polymorphism is responsible for the great interindividual difference observed in TPMT activity. An alternative hypothesis is the possibility of non-compliance. ${ }^{26}$ The frequency of non-compliance detected through 6-MP metabolite assays observed in this study is comparable to the $10 \%$ or $20 \%$ reported by British investigators. ${ }^{12-14}$ In studies which compared laboratory evaluation with interviews or health care personnel information, a satisfactory correlation between the two evaluation approaches was observed. ${ }^{6}{ }^{12} 14$ The fact that a higher number of patients have been identified as non-compliant through interviews and medical charts does not mean that metabolite assay is an inadequate method for detecting compliance. It would probably be more effective if more frequent samples were collected. Knowledge of the profile of TPMT activity could also help in the identification of non-compliant patients.

Compliance is just one variable that affects the leucocyte count in the maintenance phase of the protocol. Additionally, many counts were added to obtain a median count for each patient. It is not surprising, therefore, that no association between non-compliance and leucocyte count was found in the present study.

A significant association between malnutrition, measured by HAZ, and non-compliance was observed. A deficit in this indicator may reflect global socioeconomic shortcomings. ${ }^{17}$ Similarly there was a tendency for the monthly per capita income of the non-compliant group to be inferior to that of compliant children, which was somehow expected and compatible with other authors' findings. ${ }^{6} 71314$

The outcome of non-compliant and compliant patients seems to be different, although this may be entirely ascribed 
to chance, given that the numbers are rather small. No studies aimed at assessing the influence of compliance in the prognosis of childhood ALL were found. Although it is possible that differences in the incidence of the various subtypes of the disease might explain the disparate results obtained in ALL treatment in different countries and communities, ${ }^{27-29}$ the number of therapeutic failures is significantly higher in places where poverty, malnutrition, barriers in communication between doctors and patients, and low schooling levels prevail..$^{30}$ In certain communities and ethnic groups it is difficult to raise family awareness of the necessity of following the treatment in the maintenance phase, a time when children are apparently cured. ${ }^{731}$ All these data suggest a need for other hypotheses-aside from the biological factors-for the unfavourable prognosis of patients living in adverse conditions. The outcome of all 91 children diagnosed at UFMG during the study period was clearly inferior to that in developed countries. The estimated five year probability of disease free survival was $56.6(7.0) \%$ (unpublished data).

Information obtained in the present study can be put to the benefit of patients by mitigating the negative effects of noncompliance. There is hardly ever only one reason for the patient to disobey medical instructions. Therefore, complex interventions involving different techniques are usually necessary for obtaining satisfactory results. ${ }^{32}{ }^{33}$ In order to improve compliance, it is paramount that health care personnel understand and ponder why patients are noncompliant, what are their needs, personal priorities, and social bonds, so that their health and treatment become a priority. ${ }^{34}$

Although the sample size is insufficient for definite conclusions, the results of the present study corroborate the hypothesis that non-compliance is an important mechanism responsible for a negative impact of socioeconomic factors in the prognosis of childhood ALL. ${ }^{2}$ Results also indicate that a careful medical history, including specific questions about treatment compliance, is enough for the routine management of children with ALL; metabolite assays would be requested only in specific cases.

\section{ACKNOWLEDGEMENTS}

We are grateful to Dr Mitiko Murao, Gilberto Ramos, Cybele A Paes (Haematology Division, Hospital of Clinics, Federal University of Minas Gerais), Maria Christina Lopes Araújo Oliveira, Rosângela Carrusca Alvim, Rachel Aparecida Ferreira Femandes (Haematology Division, Department of Paediatrics, Federal University of Minas Gerais). We thank the medical graduates (Drs Carolina Oliveira Lisboa, João Baptista Horta Murta Neto, Débora Tonini Lopes, Ana Paula Oliveira Anacleto, Simone Amaral Pinheiro, Denise S Nunes, Letícia de Mattos Arruda, Mariana Inês Ybarra) who helped in the application of the questionnaires. We also thank all parents and children who participated in this study. Financial support: FAPEMIG, CPqRR-FIOCRUZ, CNPq, Pronex.

\section{Authors' affiliations}

B M de Oliveira, M B Viana, Haematology Division, Department of Paediatrics, Federal University of Minas Gerais, Belo Horizonte, Minas Gerais, Brazil

C L Zani, A J Romanha, René Rachou Research Center, Oswaldo Cruz Foundation, Belo Horizonte, Minas Gerais, Brazil

\section{REFERENCES}

1 Pui C-H, Sallan S, Relling MV, et al. International childhood acute lymphoblastic leukemia workshop: Sausalito, CA, 30 November - 1 December 2000. Leukemia 2001;15:707-15.

2 Viana MB, Fernandes RAF, Oliveira BM, et al. Nutritional and socio-economic status in the prognosis of childhood acute lymphoblastic leukemia. Haematologica 2001;86:113-20.

3 Davies HA, Lilleyman JS. Compliance with oral chemotherapy in childhood lymphoblastic leukaemia. Cancer Treat Rev 1995;21:93-103.
4 Smith SD, Rosen D, Trueworthy RC, et al. A reliable method for evaluating drug compliance in children with cancer. Cancer 1979;43:169-73.

5 Lansky SB, Smith SD, Cairns NU, et al. Psychological correlates of compliance. Am J Pediatr Hematol Oncol 1983;5:87-92.

6 Tebbi CK, Cummings KM, Zevon MA, et al. Compliance of pediatric and adolescent cancer patients. Cancer 1986;58:1179-84.

7 MacDougall LG, Wilson TD, Cohn R, et al. Compliance with chemotherapy in childhood leukemia in Africa. S Afr Med J 1989:75:481-4

8 Festa RS, Tamaroff MH, Chasalow F, et al. Therapeutic adherence to oral medication regimens by adolescents with cancer. I. Laboratory assessment. J Pediatr 1992;120:807-11

9 Snodgrass W, Smith S, Trueworthy R, et al. Pediatric clinical pharmacology of 6-mercaptopurine: lack of compliance as a factor in leukemia relapse. Proc Am Soc Clin Oncol 1984;3:204.

10 Azeemuddin S, Bharmal FM. Rapid method for evaluating compliance of 6mercaptopurine therapy in children with leukemia. J Chromatogr 1988;430:163-9

11 MacDougall LG, McElligott SE, Ross E, et al. Pattern of 6-mercaptopurine urinary excretion in children with acute lymphoblastic leukemia: urinary assays as a measure of drug compliance. Ther Drug Monit 1992;14:371-5.

12 Davies HA, Lennard L, Lilleyman JS. Variable mercaptopurine metabolism in children with leukaemia: a problem of non-compliance? BMJ 1993;306:1239-40

13 Lennard L, Welch J, Lilleyman JS. Intracellular metabolites of mercaptopurine in children with lymphoblastic leukaemia: a possible indicator of noncompliance? Br J Cancer 1995;72:1004-6.

14 Lancaster D, Lennard L, Lilleyman JS. Profile of non-compliance in lymphoblastic leukaemia. Arch Dis Child 1997;76:365-6.

15 Lau RCW, Matsui D, Greenberg M, et al. Electronic measurement of compliance with mercaptopurine in pediatric patients with acute lymphoblastic leukemia. Med Pediatr Oncol 1998;30:85-90

16 Dibley MJ, Goldsby JB, Staehling NW, et al. Development of normalized curves for the international growth reference: historical and technical considerations. Am J Clin Nutr 1987;46:736-48.

17 WHO Working Group. Use and interpretation of anthropometric indicators of nutritional status. Bull World Health Organ 1986;64:929-41.

18 Lennard L. Assay of 6-thioinosinic acid and 6-thioguanine nucleotides, active metabolites of 6-mercaptopurine, in human red blood cells. J Chromatogr 1987;423:169-78

19 Dervieux T, Boulieu R. Simultaneous determination of 6-thioguanine and methyl 6-mercaptopurine nucleotides of azathioprine in red blood cells by HPLC. Clin Chem 1998;44:551-5.

20 Lennard L, Singleton HJ. High-performance liquid chromatographic assay of the methyl and nucleotide metabolites of 6-mercaptopurine: quantitation of red blood cell 6-thioguanine nucleotide, 6-thioinosinic acid and 6methylmercaptopurine metabolites in a single sample. J Chromatogr 1992:583:83-90.

21 Dean AG, Dean JA, Coulombier D, et al. Epi info, version 6: a word processing, database, and statistics program for public health on microcomputers. Atlanta, GA: Centers for Disease Control and Prevention, 1995.

22 Wright EC. Non-compliance-or how many aunts has Matilda? Lancet 1993;342:909-13.

23 Kyngäs HA, Kroll T, Duff ME. Compliance in adolescents with chronic diseases: a review. J Adolesc Health 2000;26:379-88.

24 Matsui DM. Drug compliance in pediatrics. Pediatr Clin North Am 1997:44:1-11.

25 Cameron C. Patient compliance: recognition of factors involved and suggestions for promoting compliance with therapeutic regimens. J Adv Nurs 1996;24:244-50.

26 Relling MV, Hancock ML, Rivera GK, et al. Mercaptopurine therapy intolerance and heterozygosity at the thiopurine S-methyltransferase gene locus. J Natl Cancer Inst 1999;91:2001-8.

27 Kalwinsky DK, Rivera G, Dahl GV, et al. Variation by race in presenting clinical and biologic features of childhood acute lymphoblastic leukemia: implications for treatment outcome. Leuk Res 1985;6:817-23

28 Powell JE, Mendez E, Parkes SE, et al. Factors affecting survival in white and Asian children with acute lymphoblastic leukaemia. $\mathrm{Br} J$ Cancer 2000;82:1568-70.

29 Paes CA, Viana MB, Freire RV, et al. Direct association of socio-economic status with T-cell acute lymphoblastic leukaemia in children. Leuk Res 2003;27:789-94

30 Bonilla M, Moreno N, Marina N, et al. Acute lymphoblastic leukemia in a developing country: preliminary results of a nonrandomized clinical trial in El Salvador. Am J Pediatr Hematol Oncol 2000;22:495-501.

31 Lilleyman JS, Lennard L. Non compliance with oral chemotherapy in childhood leukaemia. BMJ 1996;313:1219-20.

32 Haynes RB, McKibbon KA, Kanani R. Systematic review of randomized trials of interventions to assist patients to follow prescriptions for medications. Lancet 1996;348:383-6.

33 Rogers AS, Miller S, Murphy DA, et al. The TREAT (Therapeutic Regimens Enhancing Adherence in Teens) Program: theory and preliminary results. J Adolesc Health 2001;29S:30-8.

34 Spinetta JJ, Masera G, Eden T, et al. Refusal, non-compliance, and abandonment of treatment in children and adolescents with cancer. A report of the SIOP working committee on psychosocial issues in pediatric oncology. Med Pediatr Oncol 2002;38:114-17.

35 Mora JO. A new method for estimating a standardized prevalence of child malnutrition from anthropometric indicators. Bull World Health Organ 1989;67:133-42 